\title{
Control and sensor techniques for PAD servo motor drive
}

\author{
Zsurzsan, Tiberiu-Gabriel; Zhang, Zhe; Andersen, Michael A. E.; Andersen, Nils Axel
}

Published in:

Proceedings of the 50th International Universities Power Engineering Conference

Link to article, DOI:

10.1109/UPEC.2015.7339868

Publication date:

2015

Document Version

Peer reviewed version

Link back to DTU Orbit

Citation $(A P A)$ :

Zsurzsan, T-G., Zhang, Z., Andersen, M. A. E., \& Andersen, N. A. (2015). Control and sensor techniques for PAD servo motor drive. In Proceedings of the 50th International Universities Power Engineering Conference IEEE. https://doi.org/10.1109/UPEC.2015.7339868

\section{General rights}

Copyright and moral rights for the publications made accessible in the public portal are retained by the authors and/or other copyright owners and it is a condition of accessing publications that users recognise and abide by the legal requirements associated with these rights.

- Users may download and print one copy of any publication from the public portal for the purpose of private study or research.

- You may not further distribute the material or use it for any profit-making activity or commercial gain

- You may freely distribute the URL identifying the publication in the public portal 


\title{
Control and sensor techniques for PAD servo motor drive
}

\author{
Gabriel T. Zsurzsan*, Zhe Zhang, Michael M.A. Andersen and Nils A. Andersen \\ Department of Electrical Engineering, Technical University of Denmark \\ Denmark \\ E-mail: *tgzsur@elektro.dtu.dk
}

\begin{abstract}
The Piezoelectric Actuator Drive (PAD) is a new type of electrical motor that employs piezoelectric multilayer actuators coupled with a form-fitted micro-mechanical gearing to generate rotary motion. The PAD is precise, having a positioning error of less than 2 arc-seconds. Its typical output torque is $4 \mathrm{~N} \cdot \mathrm{m}$, without any additional gearing. The whole motor is fully non-magnetic, enabling its use in applications where magnetic neutrality is of importance. The main challenges of the PAD are the hysteretic behavior of the ceramic actuators used and their highly capacitive nature. After compensating for the hysteretic behavior, the current waveforms of the motor can be used to extract all necessary parameters for sensorless operation. Moreover, these signals provide a qualitative information about the precision in motor centering and show any mismatch between the actuators used.
\end{abstract}

Index Terms-Piezoelectricity, multilayer actuator, motor, hysteresis, compensation, control

\section{INTRODUCTION}

$\mathbf{T}$ HE Piezoelectric Actuator Drive (PAD) is a new type of electrical rotary machine that essentially uses special ceramic actuators to impart motion. The ceramics used have piezoelectric properties - this means they physically stretch when a voltage is applied to them. This linear motion is on the order of micrometers, and is very precise.

A micro-mechanical gearing is then employed to transform the linear motion of piezoelectric actuators into a precise rotational motion [1]. These micrometer-sized teeth enable the PAD to achieve high positioning accuracy, similar to stepper motors. The motor has a positioning accuracy of less than 2 arc-seconds without the use of any positioning sensors. At the same time, the PAD achieves high typical output torques of $4 \mathrm{~N} \cdot \mathrm{m}$ without extra gearing [2]. To give a relative idea of strength, this is enough to lift a $65 \mathrm{~kg}$ sack of cement attached to a rope that is winding around the shaft.

The main advantage of using the PAD as a replacement for conventional servo drives is the fact that the PAD is a fully non-magnetic motor. This allows the PAD to be used in applications where conventional drives are inadequate, such as operation in high radiation or strong magnetic fields. One such case is operation close to the bore of a magnetic resonance imaging (MRI) scanner, as a patient table positioning motor to enable patient positioning without disturbing the image produced by the scanner itself.

Despite the advantages of the PAD, there are some challenges associated with its operation. The piezoelectric actuators used act as large capacitors, having a capacitance of $3 \mu \mathrm{F}$.
Therefore the power delivered to the motor is highly reactive. Since only the real part of the power delivered to the motor is effectively transformed into mechanical work, the power stage used to drive the motor needs to be able to cope with large amounts of return power in order to be efficient.

Furthermore, sensorless operation of the PAD is desired in order to reduce fabrication cost, improve reliability and maintain magnetic neutrality. This requires extracting information about rotor position, speed and motor torque from the motor signals without the use of expensive external sensors. Due to the stiff nature of the ceramic actuators, the load has very little influence on the motor signals and is therefore difficult to correctly estimate. Adding to this difficulty is the highly hysteretic behavior of piezoelectric ceramics in general [3]. This hysteresis needs to be compensated for in order to correctly estimate rotor position and speed.

While the micro-mechanical toothing combined with the large actuator stiffness make the PAD motor ideal for use in voltage-driven open-loop control [4], efficient operation and load-sensing capabilities can only be obtained in a closedloop control structure. This requires overcoming the aforementioned challenges.

\section{Piezoelectric STACK ACTUATORS}

Piezoelectricity has the property of being a bidirectional effect. The direct effect allows these ceramics to generate an electrical charge on their electrodes which is proportional to the force applied to their surface. The converse effect enables these materials to expand and contract a distance proportional to the voltage applied to their electrodes. The actuators employed in the motor use the converse piezoelectric effect to transform electrical energy to mechanical energy.

The range of elongation in piezoelectric ceramics is in the order of nano- to micrometers, and is therefore not inherently useful in transferring energy. Stacking many layers of piezoceramics on top of each other creates a piezoelectric multilayer actuator (PMA). These layers are connected electrically in parallel but mechanically in series, as shown in Fig. 1. The advantage of PMAs over their single layer counterparts is the ability to produce larger deflections and require a fraction of the voltage levels of its single layer counterparts. The cost comes in the form of larger exhibited capacitances and therefore higher currents for the same actuation speed. 


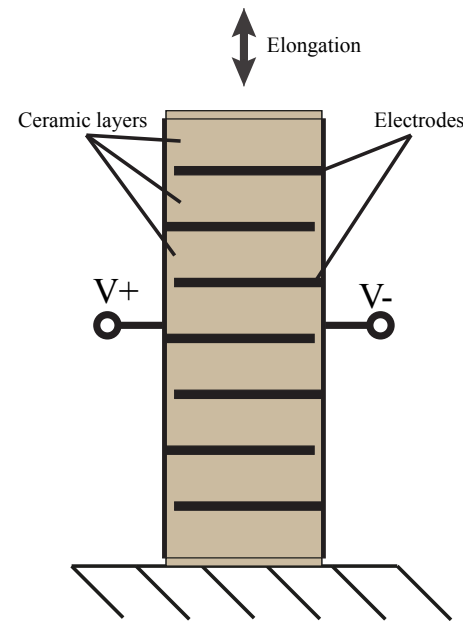

(a)

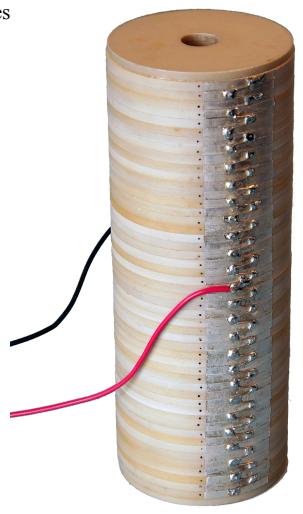

(b)
Fig. 1: Piezoelectric multilayer actuator (PMA) structure diagram (a) and actual image (b). The electrodes (black lines in (a) and silver surface with connection points in (b)) are inserted with an alternating polarity into the ceramic structure. Application of a voltage on these electrodes produces a vertical expansion of the structure.

\section{PAD OPERATING PRINCIPLE}

The PAD and its internal structure are shown in Fig. 2a and Fig. 2b. Four PMAs are placed inside the motor housing two sets of parallel actuators, with the sets having a $90^{\circ}$ angle between them. The PMAs are fixed to the motor housing on one side and to the motor ring on the other. This ring has a square exterior profile with a circular cutout in its center which houses the motor shaft. This shaft is fixed to the motor housing through ball bearings, allowing only rotation. Both the surface on the inside of the motor ring and the circumference of the shaft are machined into micro-mechanical teeth with a pitch of $100 \mu \mathrm{m}$. Fig. 2c shows a microscope image of the motor shaft teeth. The ring and shaft have 313 and 312 teeth, respectively, with the shaft having one tooth less than the ring. This forms a robust contact point between surfaces, suited for power transfer while also gives the PAD the ability to output high levels of torque through the inherent gearing ratio.

The multilayer actuators expand and contract linearly along their length and thereby push and pull against the motor ring. This creates two axes of actuation within the plane of the stack actuators, the motor ring and shaft, thereby enabling the motor ring to exhibit movement around the shaft. By applying a sinusoidal voltage to the actuators on one axis and a phaseshifted version of the same signal with a $90^{\circ}$ phase shift to the other actuators, the resulting space-vector and trajectory will be circular. Fig. 3a shows the voltages applied to the actuators normalized to their sinusoidal periods, while Fig. $3 \mathrm{~b}$ illustrates the resulting spatial trajectory. Thereby, the motor ring exhibits an off-center circular motion around the shaft which is rotated in the opposite direction through the contact point between them.

Each full period of the applied sinusoidal voltage determines the motor ring to do a full revolution around the shaft and this, in turn, makes the shaft rotate in the opposite direction for the distance equivalent to one tooth step. This is due to the inherent 312 gearing ratio present through the micromechanical toothing. Therefore a $360^{\circ}$ rotation of the contact point between the motor ring and shaft produces a $1.15^{\circ}$ rotation in the output shaft of the motor. This principle is illustrated in Fig. 4 Moreover, the frequency of the applied signals determines the speed of rotation, reduced by the same gearing ratio, such that

$$
v=\frac{N_{\text {ring }}-N_{\text {shaft }}}{N_{\text {ring }}} \cdot \frac{f}{60},
$$

where $v$ is the shaft rotation speed in RPM, $N_{\text {ring }}$ and $N_{\text {shaft }}$ represent the number of teeth on the motor ring and shaft, respectively, and $f$ is the frequency in $\mathrm{Hz}$ of the applied sinusoidal voltage signals.

\section{HYSTERESIS EFFECTS AND COMPENSATION METHODS}

As mentioned in the introduction, a disadvantage of piezoelectric ceramics is the high level of hysteresis they exhibit between the applied voltage signal and the resulting displacement. This is even more pronounced in PMAs, where the real elongation of the actuator can vary by over $20 \%$ through the middle of the stroke [5], as shown in Fig. 5a. Since hysteresis has no effect on the actual displacement amplitude of the PMAs, it does not impact open-loop positioning accuracy when motion is executed along integer number of teeth. Since one electrical revolution equals to one tooth step, a fraction of that will result in sub-tooth motion. But since this implies partial actuator expansion, this type of motion will be heavily affected by hysteresis and in turn negatively affect sub-tooth positioning accuracy. Thereby, the absolute motor accuracy is reduced significantly.

A simple way of eliminating PMA hysteresis is to measure the extent of the effect and forward-compensate for it by adapting the driving signals, as proposed in [5]. This method was proven to reduce the absolute positioning error of the actuator under test by a factor of 10 . The effects of hysteresis compensation on displacement are shown in Fig. 5a and Fig. 5b. Due to the manufacturing process of the actuators which involves chemical, thermal and polarization processes, the tolerance of their parameters is high, even for PMAs produced in the same batch. Therefore, although showing good results, the presented method requires measuring the characteristics of every actuator individually. While this might be feasible for low and very low volume manufacturing of PADs, a different method would be required for a feasible manufacturing line assembly process.

Therefore, a more feasible way of compensating for PMA hysteresis is a closed-loop approach. Methods for achieving this are currently under investigation.

\section{SENSORLESS CONTROL}

Sensorless control of electrical motors implies measuring the motor response only through its electrical signals and using these measurements to estimate all necessary motor quantities: position, speed, acceleration and torque. This allows the motor to be fully controlled without the use of external positioning or torque sensors, which represent a large part of the overall motor cost. 


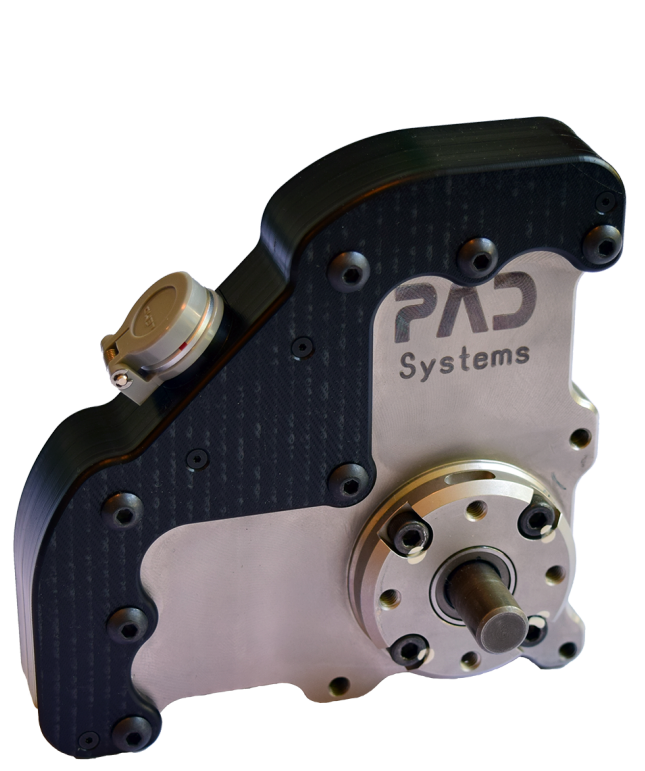

(a)

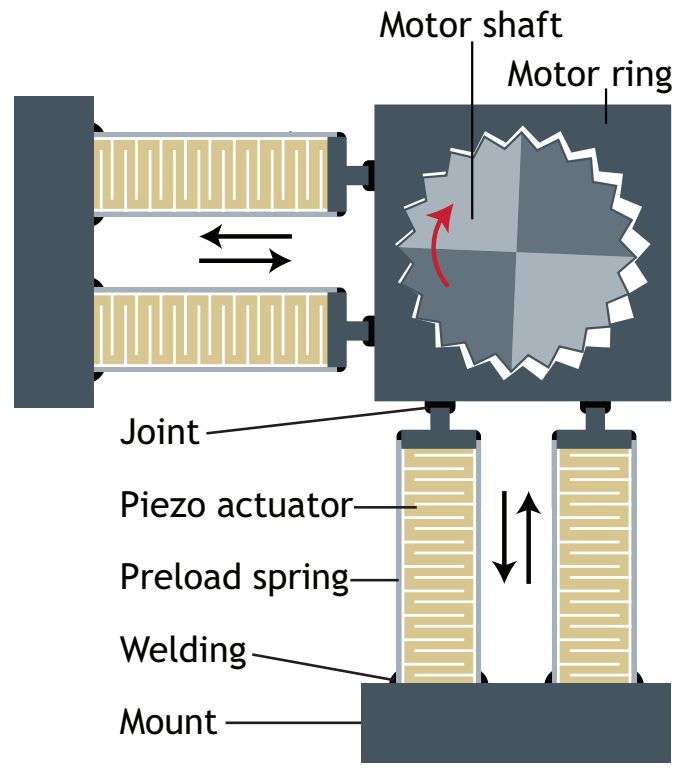

(b)

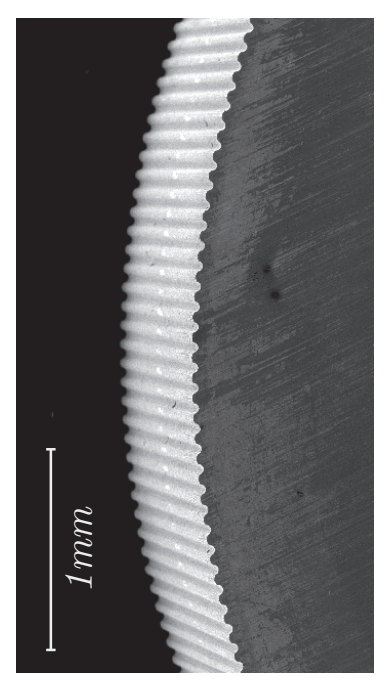

(c)

Fig. 2: PAD motor (a), internal structure (b) and micro-mechanical gearing (c). The internal structure of the PAD is made up of 4 PMA actuators acting against a motor ring, which in turn is coupled to the shaft through a micromechanical gearing. The pitch of the gears is $100 \mu \mathrm{m}$.

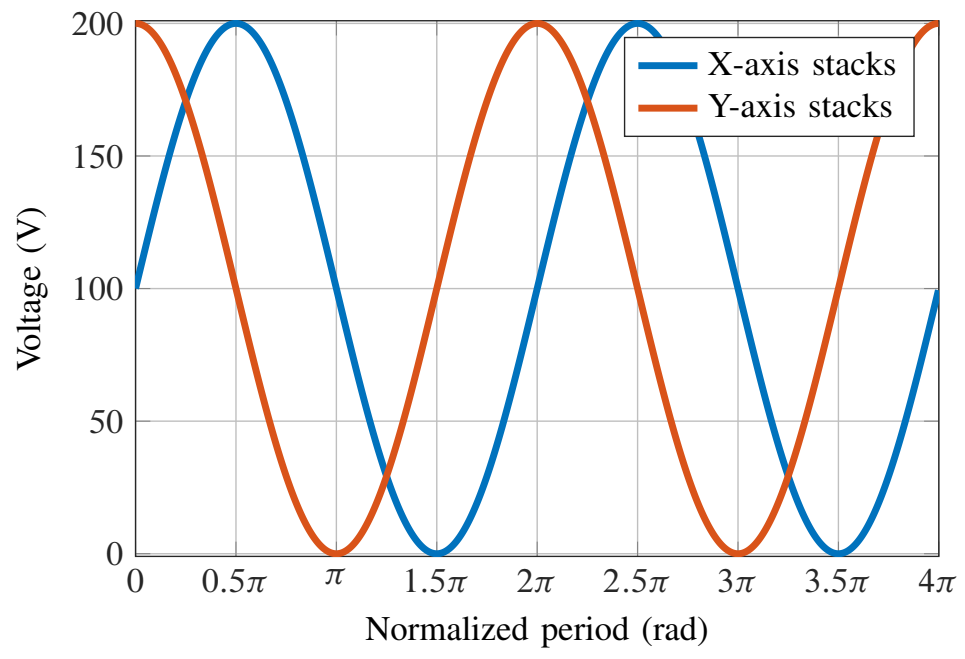

(a)

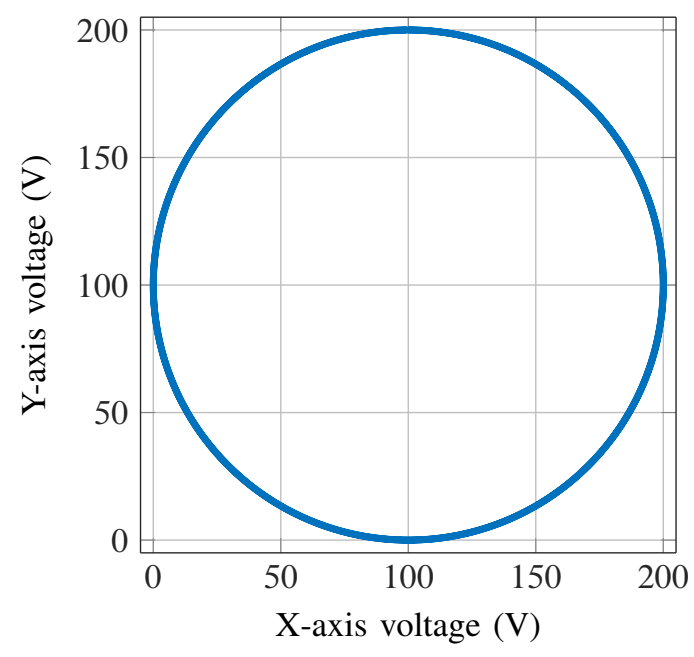

(b)

Fig. 3: Sine and cosine voltage signals applied to the actuators, normalized to their period (a) and ideal spatial trajectory obtained (b)

Due to its inherent structure, the PAD motor behaves like a fully capacitive, voltage-driven rotary machine. The piezoelectric stack actuators forming the basis of motion in this motor give it a capacitive nature. This, coupled with the reversibility of the piezoelectric effect enable the stacks to act as both actuators and sensors [6]. The current flow to and from the motor provides information about rotor speed and position, but it also indicates the quality of mechanical contact between stator and rotor through electromechanical coupling between the shaft and the stacks. In order to qualify this coupling, the motor was driven in open-loop and the current waveforms were measured and plotted against each other to form a spatial trajectory plot. Analysis of the measured data yields interesting results. Fig. 6a and Fig. 6b show the trajectory of the traveling contact point between the motor ring and shaft during a pass over 15 teeth in the rotor, under conditions of $3 \mathrm{~N} \cdot \mathrm{m}$ load and no load, respectively. Each blue curve represents one electrical revolution of the ring and one tooth step of the shaft. The red pegs each represent a rotation of one tooth on the shaft and help with visually keeping track of shaft position. The dotted black circles represent the ideal, undistorted trajectory.

These data show that position and speed can both be estimated from the measured current waveforms, although sub-tooth precision cannot be guaranteed due to hysteresis strongly affecting the measurement. Moreover, a resonance can be observed in the case of the loaded motor which cannot be seen in the unloaded case. This effect is the result of hard electromechanical coupling between the actuators and 

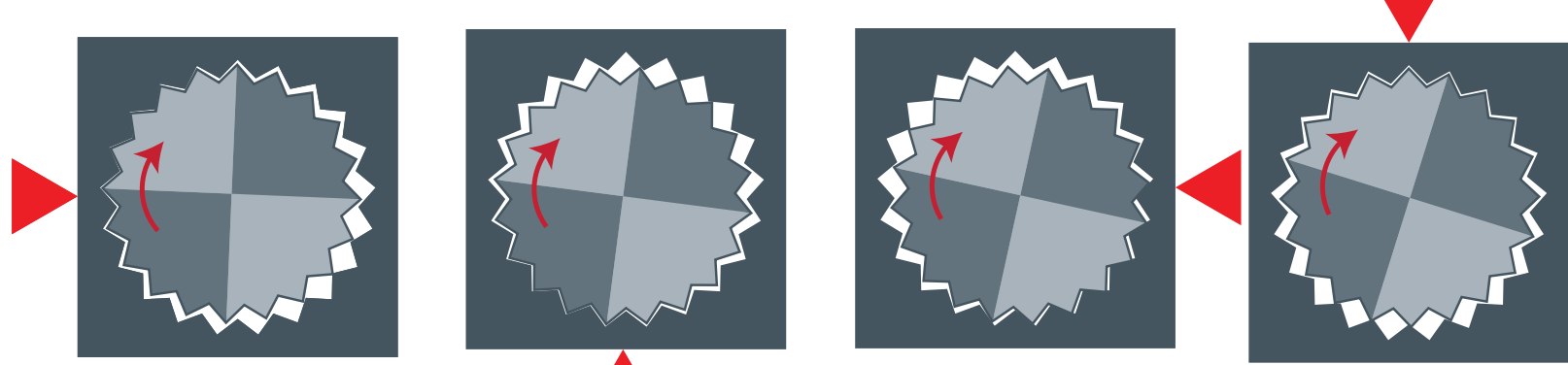

Fig. 4: The principle of rotation in the PAD motor. The red arrow represents the direction of actuation, which is the same as the position of the contact point. The shaft is fixed by a bearing while the ring is free to rotate around it. One revolution of the contact point will cause the motor shaft to turn the distance equivalent to one tooth.

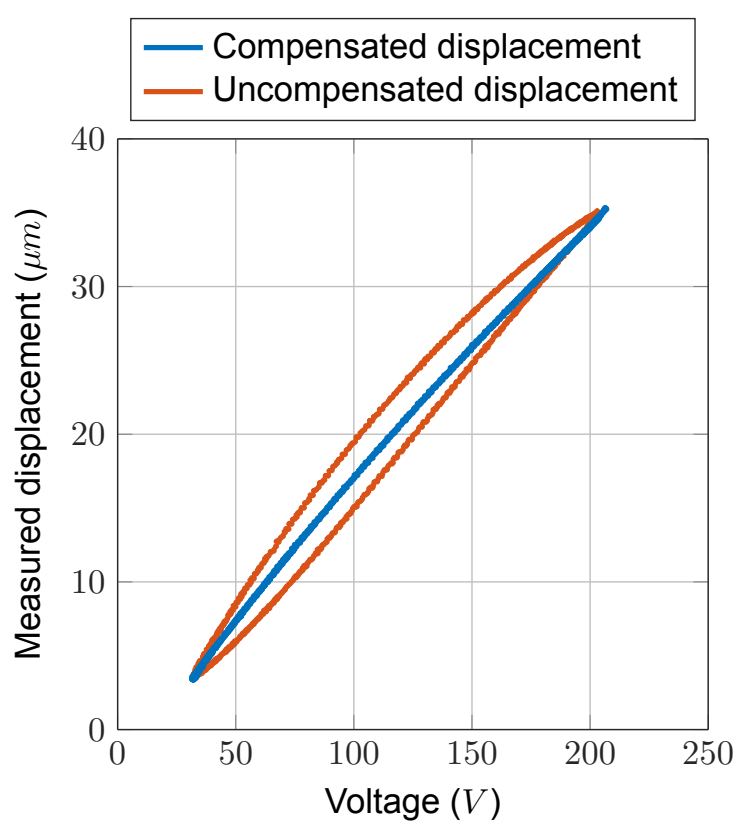

(a)

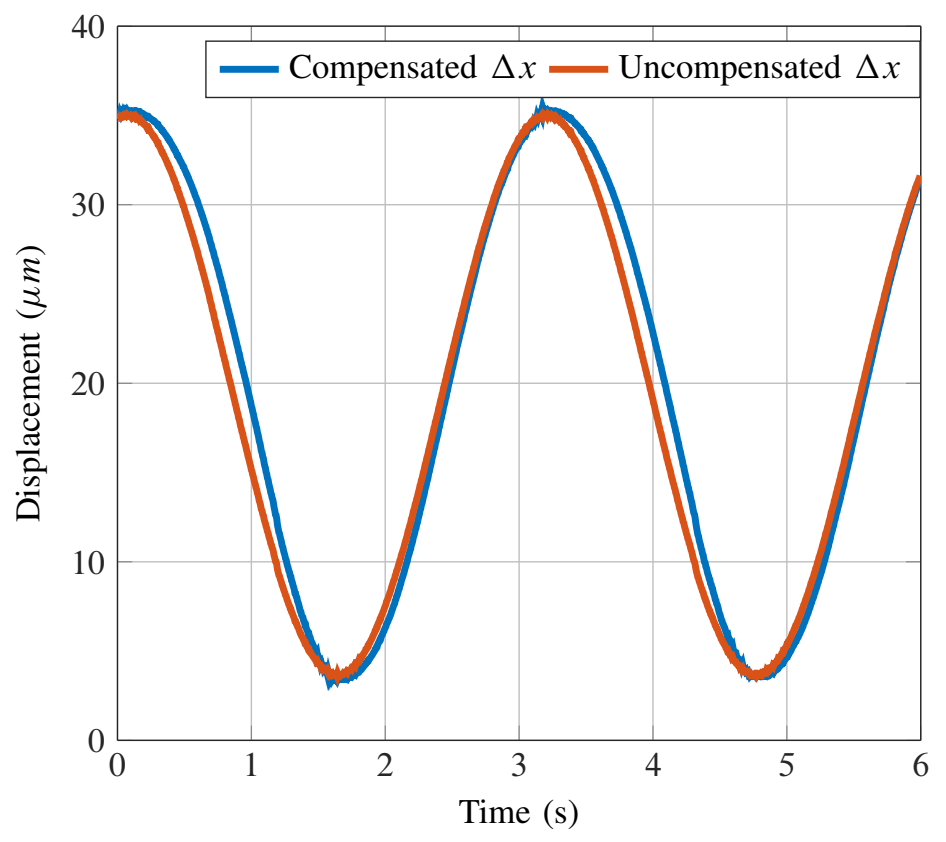

(b)

Fig. 5: Effects of hysteresis on PMA displacement versus input voltage (a), and time (b) uncompensated and compensated. Through compensation, the hysteresis exhibited between the input voltage signal and actual PMA elongation is almost fully eliminated.

the shaft, induced by the high load on the motor. This is an indication that the motor is very close to its power limit. If load or speed is increased further, a tooth skip will occur at the point of resonance.

Furthermore, a lack of symmetry along the first diagonal of the trajectory plot is observed. This indicates that the loading on the stack actuators is not symmetrical. Specifically, the flattened region visible in both the loaded and unloaded cases in the lower right area of the figures suggests a lack of proper centering between the ring and shaft. The current does not increase as it should, therefore the stacks are blocked and cannot expand properly in that quadrant.

Thus, the performed measurements provide valuable information about the motor itself without the use of any external sensors. Therefore, sensorless operation based only on the motor current is deemed possible, but only after proper compensation of any hysteretic effects. This is the next natural step in the project.

\section{Vi. Conclusion}

The Piezoelectric Actuator Drive is a rotary motor that employs the micrometer precision of piezoelectric multilayer actuators coupled with a form-fit micro-mechanical gearing in order to produce a precise, powerful rotary motion. These features, coupled with the added benefit of complete magnetic neutrality make the PAD an ideal motor to be used in harsh, radiation-heavy environments or large magnetic fields. One direct application is patient positioning in an MRI scanner where the motor has to cope with magnetic fields of up to 7 Tesla and at the same time ensure that the sensitive imaging process is not disturbed. Therefore, achieving sensorless operation is a requirement since by eliminating all external positioning and torque sensors both the cost and impact on the imaging are reduced. 


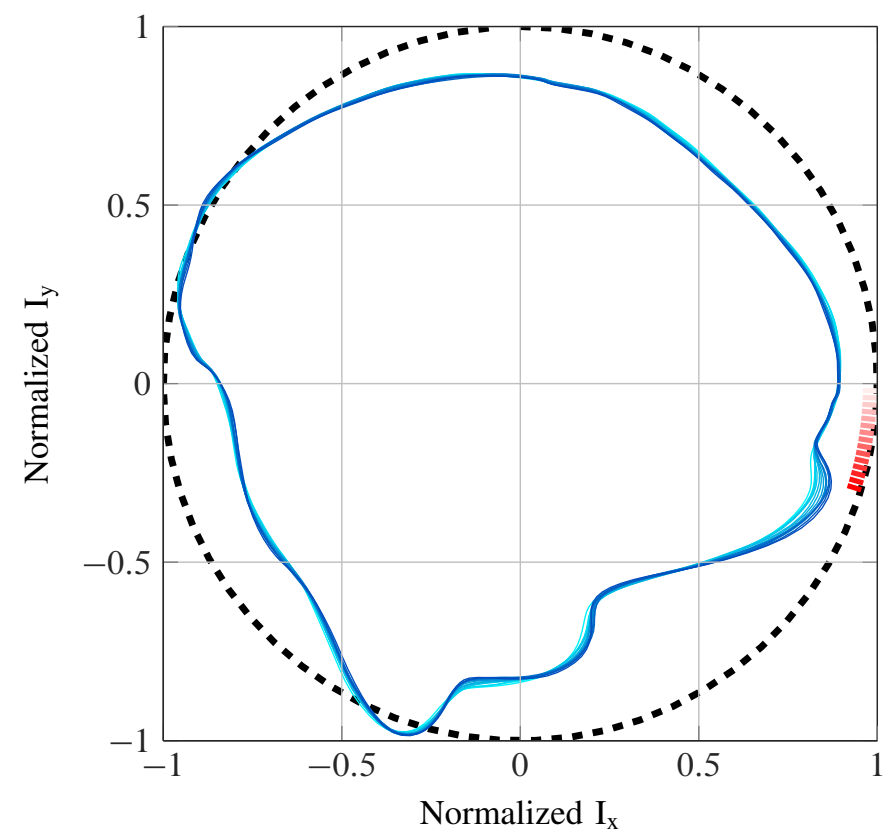

(a)

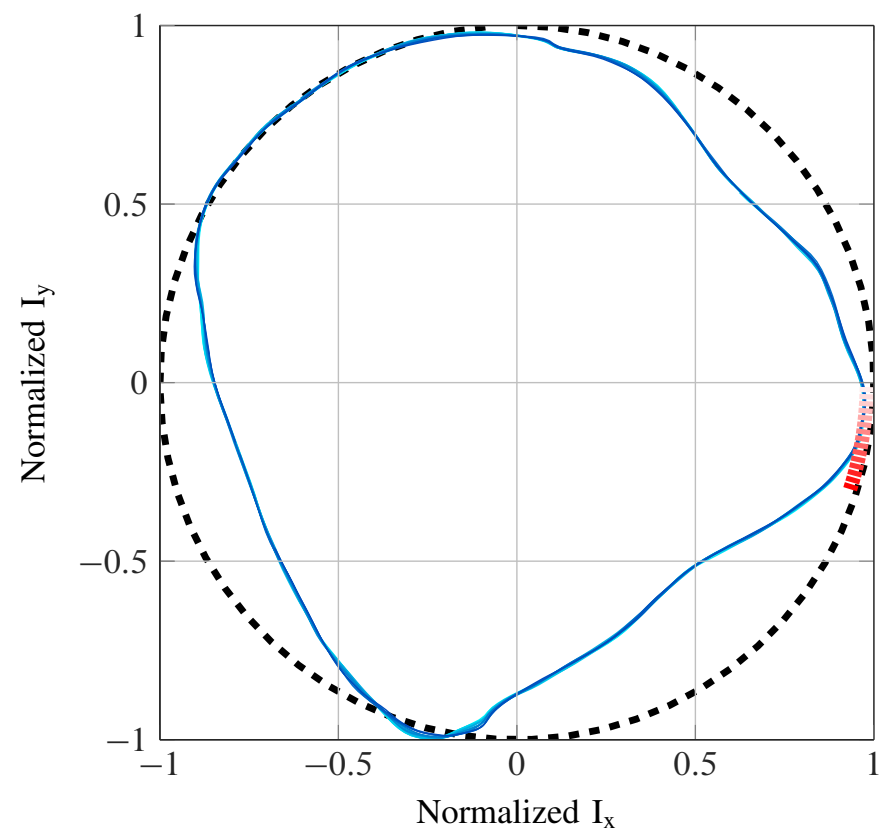

(b)

Fig. 6: Normalized contact point trajectory plot under load (a) and no load (b). In both images, the black dashed circle represents the ideal, desired trajectory. Dark blue and dark red denote a more recent revolution and tooth step, while lighter colors are used to represent the 'history' of the trajectory point in time.

A first step in improving the dynamic operation of the motor is to compensate for the hysteretic behavior of the PMAs, which form the basis of the motion in the motor. Some good results have been achieved through precompensation, but this method is limited in practicality and therefore a feedbackbased compensation method is being investigated.

The preliminary analysis of the information contained in the motor current waveforms shows the quality of the mechanical contact point between the motor ring and shaft. A first observation is the major impact PMA hysteresis has on the measurements performed. Nonetheless, the trajectory plot of the ring-shaft contact point extracted from the normalized current measurements indicate rotor-ring misalignment as well as show the absolute position of the weakest point of contact between the two.

Future work will encompass a full removal of all the effects of hysteresis, followed by a new set of current measurements in order to better analyze the behavior of the actuators. This will eventually lead to estimating the desired motor quantities - position, speed and torque - from the measured current waveforms and thereby achieving closed-loop sensorless operation.

\section{ACKNOWLEDGEMENTS}

The authors would like to thank Noliac A/S for providing a PAD motor to be dissected and investigated, as well as the Danish National Advanced Technology Foundation for financial support.

\section{REFERENCES}

[1] A. Kappel, B. Gottlieb, and C. Wallenhauer, "Piezoelectric actuator drive (pad)," AT-AUTOMATISIERUNGSTECHNIK, vol. 56, no. 3, pp. 128-135, 2008.

[2] J. Rucha, H. Liess, J. Heinzl, A. Kappel, T. Schwebel, and B. Gottlieb, "Applicability of a piezo actuator drive as a sensorless high precision drive," 10th International Conference on New Actuators, 2006.

[3] "Standard on piezoelectricity," IEEE, 1988.

[4] R. Zeichfussl, B. Gottlieb, C. Wallenhauer, S. Herzig, A. Kappel, and T. Luth, "A method for auto-adjustment of a new piezoelectric drive," in Mechatronics, 2009. ICM 2009. IEEE International Conference on, April 2009, pp. 1-6.

[5] G. T. Zsurzsan, C. Mangeot, M. A. E. Andersen, Z. Zhang, and N. A Andersen, "Piezoelectric stack actuator parameter extraction with hysteresis compensation," Power electronics and applications, 16th European Conference on, in press.

[6] C. Wallenhauer, B. Gottlieb, A. Kappel, T. Schwebel, J. Rucha, and T. Lueth, "Accurate load detection based on a new piezoelectric drive principle employing phase-shift measurement," Journal of Microelectromechanical Systems, vol. 16, no. 2, pp. 344-350, 2007. 